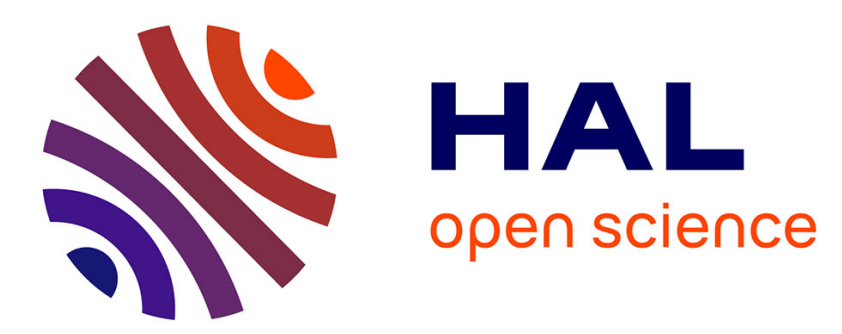

\title{
Méthode d'étude des fluctuations de potentiel local et de densité dans un plasma alcalin
}

\author{
J.R. Cussenot, M. Felden
}

\section{To cite this version:}

J.R. Cussenot, M. Felden. Méthode d'étude des fluctuations de potentiel local et de densité dans un plasma alcalin. Revue de Physique Appliquée, 1969, 4 (4), pp.519-524. 10.1051/rphysap:0196900404051900 . jpa-00243323

\section{HAL Id: jpa-00243323 https://hal.science/jpa-00243323}

Submitted on 1 Jan 1969

HAL is a multi-disciplinary open access archive for the deposit and dissemination of scientific research documents, whether they are published or not. The documents may come from teaching and research institutions in France or abroad, or from public or private research centers.
L'archive ouverte pluridisciplinaire HAL, est destinée au dépôt et à la diffusion de documents scientifiques de niveau recherche, publiés ou non, émanant des établissements d'enseignement et de recherche français ou étrangers, des laboratoires publics ou privés. 


\title{
MÉTHODE D'ÉTUDE DES FLUGTUATIONS DE POTENTIEL LOGAL ET DE DENSITÉ DANS UN PLASMA ALGALIN
}

\author{
Par J. R. GUSSENOT et M. FELDEN, \\ Laboratoire de Physique des Milieux Ionisés, 2, rue de la Citadelle, 54-Nancy, or. \\ (Reçu le 2.j juin 1969, révisé le 1er septembre 1969.)
}

\begin{abstract}
Résumé. - La mesure des paramètres fluctuants d'un plasma ayant des oscillations de densité ionique et électronique et de potentiel local est étudiée à partir de la connaissance des composantes perturbées et non perturbées du courant collecté par une sonde à grille. Après une rapide analyse théorique de la méthode utilisée, des expériences de vérification sont faites dans un plasma de césium. Ces résultats sont en bon accord avec les prévisions théoriques.
\end{abstract}

Abstract. - The fluctuations measurement of a plasma having parameters oscillations such as space potential, ion and electron densities is made from the knowledge of the disturbed and no disturbed currents collected by a grid probe. After a brief theoretical method analysis, experiments are made in a caesium plasma, the aim being to investigate the theoretical purpose. Experimental results are in a good agreement with the theoretical part.

Introduction. - Le potentiel local, les densités et températures des ions et électrons et les fonctions de distribution énergétique correspondantes, peuvent être étudiés de différentes manières, principalement par les sondes électriques de Langmuir [1]. Cependant, on ne peut généralement obtenir que la valeur moyenne de ces paramètres et, s'ils présentent des instabilités, leur mesure est très difficile à réaliser. Cette étude a pour but l'analyse des composantes fluctuantes du potentiel local et des densités, les mesures étant faites avec une sonde à grille.

Fonctionnement de la sonde à grille. - Cette sonde a été décrite dans les références [2-3-4]. Rappelons qu'elle est pourvue d'une grille métallique à mailles très fines qui est polarisable depuis l'extérieur de l'enceinte à plasma. Les charges doivent la traverser avant d'atteindre l'électrode de collection. Il existe deux modes de fonctionnement :

- dans le premier, la grille fait la séparation électrostatique des charges, la sélection énergétique étant réalisée par l'électrode placée derrière,

- dans le second, c'est l'inverse qui est réalisé.

C'est ce dernier cas que nous utilisons et, la théorie de Langmuir lui étant applicable [4-5], nous allons brièvement en rappeler le principe. Le collecteur de la sonde est porté à un potentiel $V_{c}$ très positif ou très négatif par rapport au potentiel de plasma $V_{\mathrm{p}}$ selon que l'on veuille étudier uniquement les électrons ou les ions. Le potentiel de grille $V_{\mathrm{g}}$ varie au voisinage du potentiel local $V_{\mathrm{p}}$, à $V_{\mathrm{g}}-V_{\mathrm{c}}=$ Cte, ce qui permet la sélection en énergie. Le courant $I_{\mathrm{s}}^{\varepsilon}$ collecté par la sonde dépend de $V_{\mathrm{S}}=V_{\mathrm{g}}-V_{\mathrm{p}} \quad(\varepsilon= \pm 1$ représente le signe des charges) :

$$
I_{\mathrm{s}}^{\varepsilon}=0
$$

quand toutes les charges sont repoussées,

$$
I_{\mathrm{S}}^{\varepsilon}=S_{e} N_{\varepsilon}\left(\frac{K T_{\varepsilon}}{2 \Pi m_{\varepsilon}}\right)^{1 / 2} \exp \left(\frac{-\varepsilon e V_{\mathrm{S}}}{K T_{\varepsilon}}\right)
$$

pour une distribution maxwellienne.

La surface de collection est représentée par $S, e$ est la charge élémentaire, $K$ la constante de Boltzmann, $N_{\varepsilon}, T_{\varepsilon}$ et $m_{\varepsilon}$ indiquent respectivement la densité, la température et la masse des charges de type $\varepsilon$ :

$$
I_{\mathrm{S}}^{\varepsilon}=\operatorname{SeN}_{\varepsilon}\left(\frac{K T_{\varepsilon}}{2 \Pi m_{\varepsilon}}\right)^{\mathbf{1} / 2}
$$

pour la collection à saturation à travers la charge propre d'espace.

Dans tous les cas, on a :

$$
I_{\mathrm{s}}^{\varepsilon}=\operatorname{SeN} N_{\varepsilon} g\left(V_{\mathrm{S}}\right)
$$

où $g\left(V_{\mathrm{S}}\right)$ est une fonction génératrice dépendant de $V_{\mathrm{s}}$.

Mesure des paramètres fluctuants d'un plasma. Plaçons-nous dans le cas où seules existent des fluctuations de densité et de potentiel local, celles de température étant supposées négligeables [6], soient :

$$
\begin{gathered}
N=N_{0}+n_{1} \mathrm{e}^{j(\omega t+\varphi)} \\
V_{\mathrm{s}}=V_{\mathrm{s}_{0}}+v_{\mathrm{s}_{1}} \mathrm{e}^{j \omega t}
\end{gathered}
$$

les expressions des densités et potentiels en fonction du temps, $\omega$ étant la pulsation et $\varphi$ le déphasage rela- 
tif, le courant instantané collecté par la sonde a pour valeur :

$$
I_{\mathrm{S}}=S e\left[N_{0}+n_{1}(t)\right] g\left[V_{\mathrm{S}_{0}}+v_{\mathrm{S}_{1}}(t)\right]=I_{\mathrm{S}_{0}}+i_{1}(t)
$$

$I_{\mathrm{S}_{0}}$ étant la valeur moyenne du courant en l'absence de fluctuation et $i_{1}(t)$ la composante fluctuante. Sur un intervalle de temps $\tau$, on a :

$$
\begin{aligned}
\overline{I_{\mathrm{S}}}=\overline{I_{\mathrm{S}_{0}}} & +i_{1}(t) \\
& =\frac{1}{\tau} \int_{0}^{\tau} S e\left[N_{0}+n_{1}(t)\right] g\left[V_{\mathrm{S}_{0}}+v_{\mathrm{S}_{1}}(t)\right] \mathrm{d} t .
\end{aligned}
$$

Pour un potentiel retardateur dans le cas d'une distribution maxwellienne avec une fluctuation du potentiel d'espace correspondant à une variation d'énergie $e v_{\mathrm{S}_{1}}$ petite devant $K T_{\varepsilon}$, on obtient [6] :

$$
\frac{\overline{I_{\mathrm{S}}}}{I_{\mathrm{S}_{0}}}=1+\frac{1}{4}\left(\frac{e v_{\mathrm{S}_{1}}}{K T_{\varepsilon}}\right)^{2}+\frac{e v_{\mathrm{S}_{1}} n_{1 \varepsilon}}{2 N_{0 \varepsilon} K T_{\varepsilon}} \cos \varphi
$$

relation montrant qu'il y a une augmentation du courant par suite de l'existence des fluctuations. Remarquons que dans cette dernière relation les variations de la composante continue sont au second ordre par rapport aux perturbations de densité et de potentiel. Il est plus intéressant de trouver des relations faisant intervenir ces perturbations au premier ordre.

Si la sonde collecte à saturation, il n'y a aucune variation de courant moyen $\frac{\overline{I_{\mathrm{S}}}}{I_{\mathrm{S}_{0}}}=1$.

Pour le courant électronique, la courbe théorique donnant $\log \overline{I_{\mathrm{S}}}=f\left(V_{\mathrm{g}}-V_{\mathrm{p}}\right)$ du plasma perturbé ou non est la suivante (fig. 1).

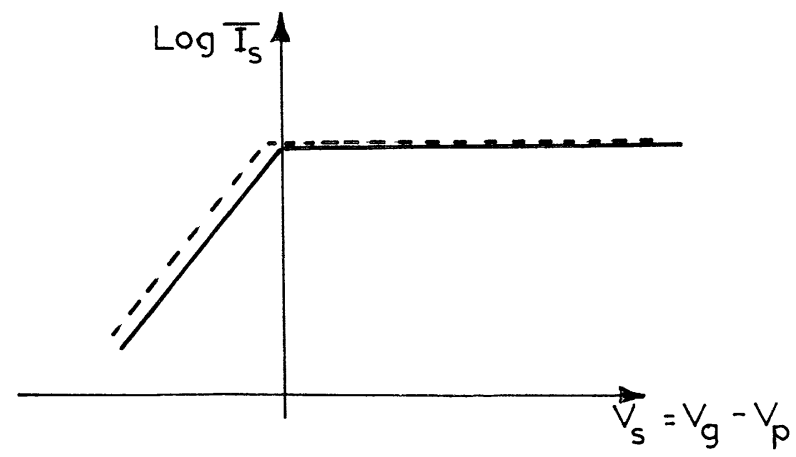

FIG. 1. - Variation du courant moyen collecté par une sonde à grille :

- Sans oscillations

- - - Avec oscillations.

Cependant, la connaissance de $\overline{I_{\mathrm{S}}}$ ne donne qu'une relation entre les deux quantités à déterminer $n_{1 \varepsilon}$ et $v_{\mathrm{S}_{1}}$. Pour en obtenir une deuxième, on peut étudier la variation de l'amplitude du courant fluctuant $i(t)$ collecté par la sonde en fonction de la tension appliquée.

La relation (1) devient, en présence d'oscillations :

$$
I_{\mathrm{S}}=S e\left[N_{0}+n_{1} \mathrm{e}^{j(\omega t+\varphi)}\right] g\left[V_{\mathrm{S}_{0}}+v_{\mathrm{S}_{1}} \mathrm{e}^{j \omega t}\right]
$$

un développement de Taylor au 1 er ordre donne :

d'où :

$$
\begin{aligned}
I_{\mathrm{S}}=S e N_{0} g\left(V_{\mathrm{S}_{0}}\right)+S e N_{0} v_{\mathrm{S}_{1}} g^{\prime}\left(V_{\mathrm{S}_{0}}\right) \mathrm{e}^{j \omega t} \\
+\operatorname{Sen}_{1} g\left(V_{\mathrm{S}_{0}}\right) \mathrm{e}^{j(\omega t+\varphi)} .
\end{aligned}
$$

L'amplitude $i$ du courant collecté est donnée par la relation :

$$
i=\operatorname{Se} N_{0} v_{\mathrm{S}_{1}} g^{\prime}\left(V_{\mathrm{S}_{0}}\right)+\operatorname{Sen} n_{1} g\left(V_{\mathrm{S}_{0}}\right) \mathrm{e}^{j \varphi} .
$$

Le premier terme est proportionnel à la fluctuation de potentiel et le second à la fluctuation de densité, $\varphi$ représentant le déphasage relatif. Pour une sonde collectant à saturation, le premier terme étant nul $g^{\prime}\left(V_{\mathrm{S}_{0}}\right)=0$, la phase relative n'intervient plus et l'amplitude a pour expression :

$$
\left(\frac{i}{I_{\mathrm{S}_{0}}}\right)_{\mathrm{sat}}=\frac{n_{1}}{N_{0}}
$$

Si elle sélectionne les particules en énergie, dans le cas d'une distribution maxwellienne :

$$
\left(\frac{i}{I_{\mathrm{S}_{0}}}\right)_{\mathrm{sel}}=\frac{n_{1 \varepsilon}}{N_{0 \varepsilon}} \mathrm{e}^{j \varphi}-\frac{\varepsilon e v_{\mathrm{S}_{1}}}{K T_{\varepsilon}}
$$

Les relations $(6)$ et $(7)$ donnent $n_{1}$ et $v_{\mathrm{S}_{1}}$, mais il reste à déterminer la phase $\varphi$ entre les variations de $n_{1}$ et $v_{\mathrm{S}_{1}}$. Ceci peut être réalisé expérimentalement, comme indiqué dans la référence [10]. Brièvement, le principe est le suivant : on utilise un détecteur synchrone ayant comme tension de référence de phase la tension excitatrice de perturbation de plasma, ce qui permet d'étudier la projection, sur un plan variable $p$, du courant fluctuant collecté par la sonde : désignons par $\mathbf{i}_{1}\left(v_{\mathrm{S}_{1}}\right)$ le vecteur ayant pour module $S e N_{0} v_{\mathrm{S}_{1}} g^{\prime}\left(V_{\mathrm{S}_{0}}\right)$ et par $\mathbf{i}_{2}(n)$ le vecteur de module $\operatorname{Sen}_{1} g\left(V_{\mathrm{S}_{0}}\right)$. Ces deux vecteurs sont déphasés l'un par rapport à l'autre de l'angle $\varphi$. Étudions leurs projections sur le plan $p$; dans le cas où $\mathbf{i}_{2}(n)$ est perpendiculaire au plan de projection, il vient :

$$
\operatorname{proj}\left[\mathbf{i}_{1}\left(v_{\mathrm{S}_{1}}\right)\right]=\operatorname{SeN} N_{0} g^{\prime}\left(V_{\mathrm{S}_{0}}\right) v_{\mathrm{S}_{1}} \cos \left(\mathbf{i}_{1}, \mathbf{p}\right)
$$

et si $\mathbf{i}_{1}\left(v_{\mathrm{S}_{1}}\right)$ est perpendiculaire à $p$ :

$$
\operatorname{proj}\left[\mathbf{i}_{2}(n)\right]=\operatorname{Sen}_{1} g\left(V_{\mathbf{S}_{0}}\right) \cos \left(\mathbf{i}_{2}, \mathbf{p}\right)
$$

le déphasage entre ces deux vecteurs étant égal à $\varphi=\left|\left(\mathbf{i}_{2}, \mathbf{p}\right)-\left(\mathbf{i}_{1}, \mathbf{p}\right)\right|$.

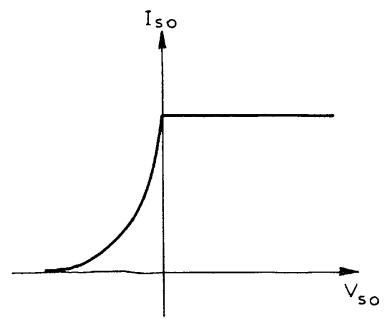

a

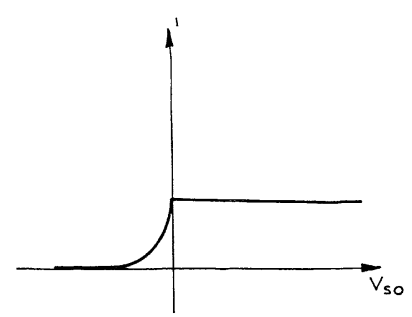

$\mathrm{b}$
FIG. 2. - Variations des courants $I_{\mathrm{S}_{0}}$ et $i$ dans le cas d'une fluctuation de densité. 
Si nous n'avons qu'une fluctuation de densité, l'amplitude $i$ est proportionnelle à la courbe $I_{\mathrm{S}_{0}}=f\left(V_{\mathrm{S}_{0}}\right)$ comme le montrent les figures $2 \mathrm{a}$ et $2 \mathrm{~b}$.

Si le potentiel fluctue seul, $i$ est proportionnel à $v_{\mathrm{S}_{1}}$ et à la dérivée première de la courbe caractéristique directe $I_{\mathrm{S}_{\mathrm{o}}}=f\left(V_{\mathrm{S}_{\mathrm{o}}}\right)$, ce qu'indique la figure 3 .

En présence de deux types de fluctuations, on a la figure 4 .

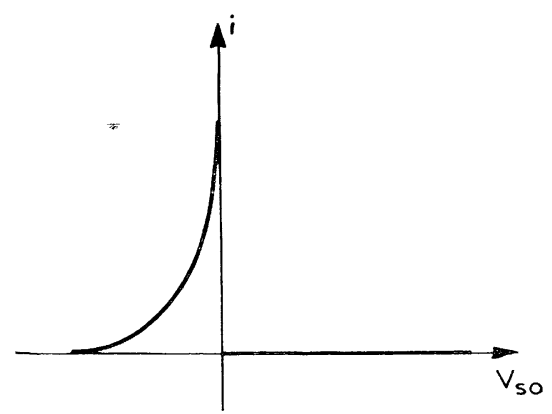

FIG. 3. - Variation du courant $i$ dans le cas d'une simulation de potentiel.

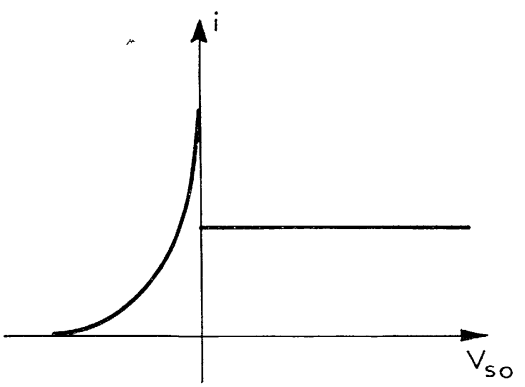

FIG. 4. - Variation de $i$

dans le cas de fluctuation de densité et du potentiel.

Pour que cette théorie soit valable, il faut limiter le développement, dans la relation (5), au premier ordre, soit, pour une distribution maxwellienne, il faut que :

$$
e v_{\mathrm{S}_{1}} \ll K T_{\varepsilon} .
$$

Etude expérimentale. - Les expériences sont réalisées dans un plasma alcalin, obtenu par une décharge à cathode chaude dans une vapeur saturante de césium [7-8]. Les différents paramètres (température de la cathode, pression de césium et différence de potentiel entre les électrodes) sont choisis pour obtenir un plasma suffisamment stable, la tension de bruit entre anode et cathode étant inférieure à quelques millivolts.

Nous avons vu dans la théorie que, pour calculer la valeur des fluctuations de densité et de potentiel, il fallait connaître les courbes caractéristiques $I_{\mathrm{S}_{0}}=f\left(V_{\mathrm{S}_{0}}\right)$ et $i=h\left(V_{\mathrm{S}_{0}}\right)$ qui donnent le courant non perturbé $I_{\mathrm{S}_{0}}$ et l'amplitude du courant oscillant $i$, en fonction de la tension de sonde. La méthode expérimentale d'enregistrement est décrite dans la référence [9].

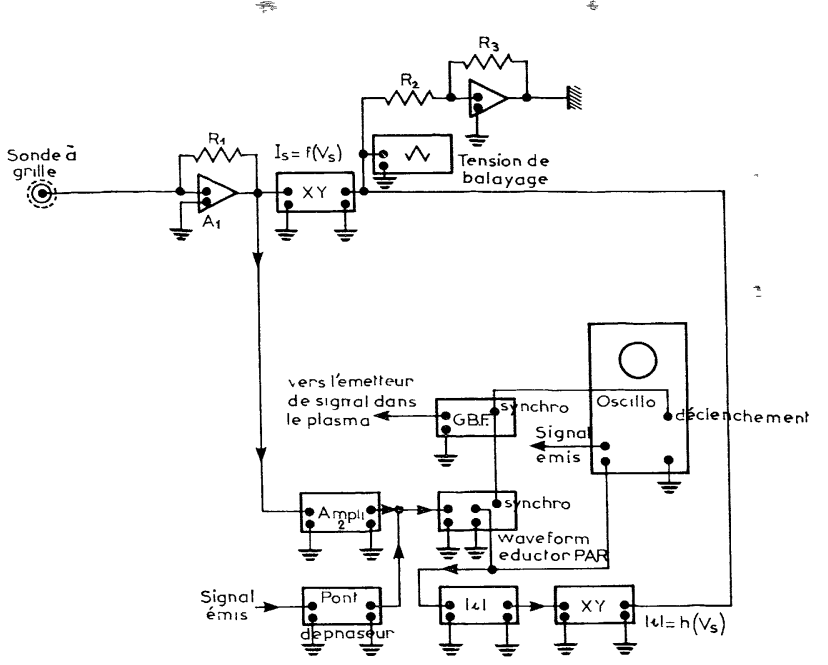

FIG. 5. - Schéma du circuit de mesure utilisé pour l'étude des oscillations de plasma.

Pour obtenir $i=h\left(V_{\mathrm{S}_{0}}\right)$, nous utilisons le circuit constitué par (fig. 5) :

- une sonde à grille décrite en référence [4],

- un amplificateur $\mathrm{A}_{1}$ à large produit « gain $\times$ bande passante » qui donne une tension proportionnelle au courant,

- un amplificateur de tension $A_{2}$ pour amplifier suffisamment la composante sinusoïdale,

- un waveform Eductor P.A.R. permettant d'extraire un signal périodique du bruit de fond. Son fonctionnement est synchronisé avec celui du générateur de perturbations B.F. Le signal, mis en mémoire dans l'appareil, est envoyé sur l'entrée d'un oscilloscope dont le balayage est synchronisé avec le générateur B.F. Gette visualisation du courant fluctuant nous permet de suivre les variations de phase du courant fluctuant ainsi que celle de l'amplitude,

- dans le cas où l'on veut mesurer le déphasage $\varphi$ entre les deux perturbations de densité et de potentiel, on remplace la waveform Eductor par un détecteur synchrone (Lock-In Amplifier P.A.R.),

- un pont déphaseur est utilisé pour compenser un courant parasite d'origine capacitive. En effet, il existe entre la sonde et le plasma une capacité c de l'ordre de $10^{-12} \mathrm{~F}$ qui est à l'origine d'un courant $i_{\text {cap }}=j C \omega v_{\mathrm{S}_{1}} \mathrm{e}^{j \omega t}$, indépendant de la polarisation continue de la sonde. L'expression de l'amplitude du courant fluctuant collecté par la sonde devient :

$i=j C \omega v_{\mathrm{S}_{1}}+S e N_{0 \varepsilon} v_{\mathrm{S}_{1}} g^{\prime}\left(V_{\mathrm{S}_{0}}\right)+S e n_{1 \varepsilon} g\left(V_{\mathrm{S}_{0}}\right) \mathrm{e}^{j \varphi}$ lorsqu'il n'y a aucune collection de charge $g\left(V_{\mathrm{S}_{0}}\right)=g^{\prime}\left(V_{\mathrm{S}_{0}}\right)$, le courant capacitif est seul mesuré. On le compense par la superposition d'un autre courant en opposition de phase et de même amplitude. Ce phénomène intervient surtout dans 
le cas d'une collection ionique, les courants capacitifs et les courants de perturbation étant du même ordre de grandeur. Dans le cas d'une collection électronique, les effets capacitifs sont négligeables devant les autres courants. Si, dans certaines conditions, il existe un flux d'électrons primaires, son effet s'ajoute aux fluctuations observées sur le milieu,

- un enregistreur XY, placé derrière un analyseur d'amplitude pour signal sinusoïdal, permet de tracer la courbe donnant la valeur absolue $|i| \mathrm{du}$ courant oscillant en fonction de la polarisation de sonde. Ce sont celles qui sont reproduites dans la partie expérimentale.

Simulation d'oscillations de potentiel. - Nous savons que l'expression du courant collecté par une sonde dépend de la différence de potentiel $V_{\mathrm{s}}=V_{\mathrm{g}}-V_{\mathrm{p}}$. En conséquence, la fluctuation de $V_{\mathrm{p}}$ peut être simulée par celle de $V_{\mathrm{g}}$ en superposant à la tension de grille un petit signal $v_{\mathrm{S}_{1}} \mathrm{e}^{j \omega t}$. Dans ce cas, si les dimensions de la sonde et l'amplitude $v_{\mathrm{S}_{1}}$ sont suffisamment faibles pour donner une faible fluctuation du plasma, nous n'observons aucune oscillation de densité [6], nous avons alors :

$$
I_{\mathrm{S}_{0}}=\operatorname{SeN} N_{0 \varepsilon} g\left(V_{\mathrm{S}_{0}}\right)
$$

les variations théoriques et expérimentales étant représentées par les figures 2 a et 6 a,

$$
i=S e N_{0 \varepsilon} v_{\mathrm{S}_{1}} g^{\prime}\left(V_{\mathrm{S}_{0}}\right)
$$

dont les variations théoriques et expérimentales sont portées sur les figures 3 et $6 \mathrm{~b}$.

Rappelons notamment que, dans la partie de sélection des charges en énergie par la grille, nous devons vérifier les relations suivantes :

$$
\left(\frac{i}{I_{\mathrm{S}_{0}}}\right)_{\text {sel }}=\frac{e v_{\mathrm{S}_{1}}}{K T_{\varepsilon}} \quad \text { et } \quad \frac{e v_{\mathrm{S}_{1}}}{K T_{\varepsilon}} \ll 1 .
$$

Les figures expérimentales 6 a et $6 \mathrm{~b}$, obtenues dans le cas d'une collection électronique seule, donnent la variation de $I_{\mathrm{S}_{0}}$ et de $i$ en fonction de la différence de potentiel $V_{\mathrm{S}_{0}}$.

Si la courbe $i=h\left(V_{\mathrm{S}_{0}}\right)$ présente bien le pic caractéristique de dérivation, il existe des différences entre la théorie et l'expérience.

- Quand $V_{\mathrm{S}} \ll 0$, la sonde collecte un courant non nul; celui-ci est dû à un flux d'électrons primaires issus de la cathode et ayant une énergie de plusieurs électron-volts.

- Quand $V_{\mathrm{S}}>0$, la théorie de la sonde plane infinie prévoit un courant à saturation constant; dans le cas présent, la sonde est de dimension finie et elle collecte un courant $I_{\mathrm{S}_{0}}$ qui augmente de façon sensiblement linéaire; dans cette région, l'amplitude $i$ est alors constante. Ce courant est dû uniquement à une fluctuation du potentiel et a une amplitude proportionnelle à la dérivée première $g^{\prime}\left(V_{\mathrm{S}_{0}}\right)$ et à $v_{\mathrm{S}_{1}}$.
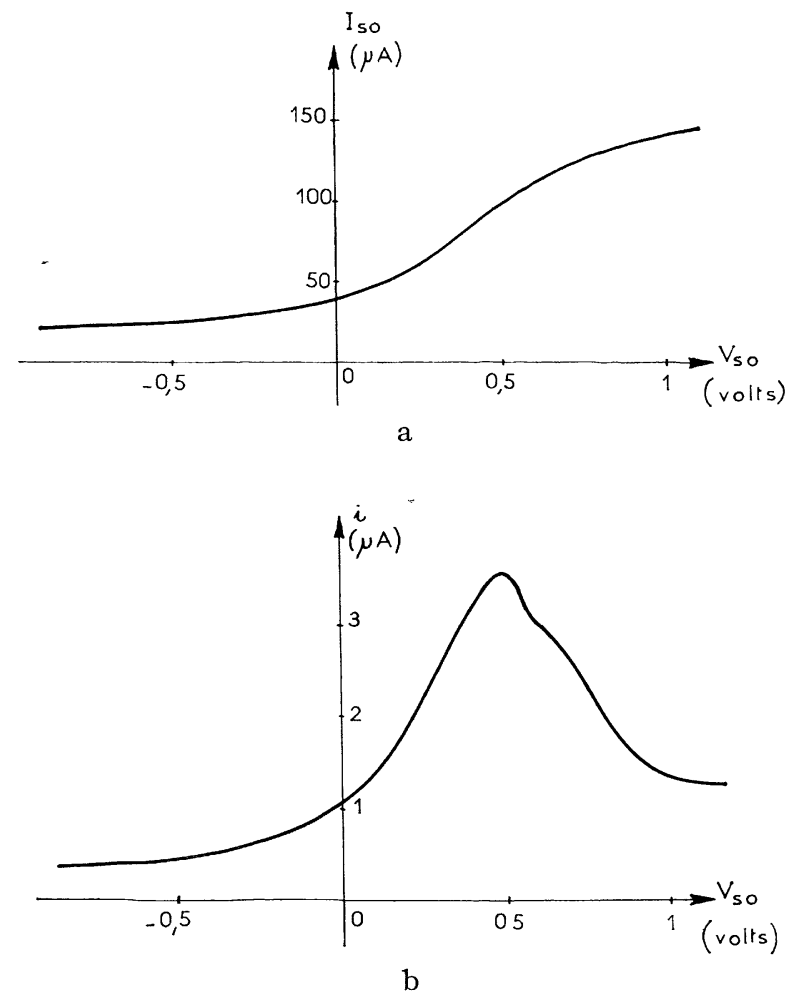

FIG. 6. - Variations expérimentales des courants $I_{\mathrm{S}_{\mathbf{0}}}$ ( fig. 6 a) et $i$ (fig. $6 \mathrm{~b}$ ), dans le cas d'une simulation d'oscillations de potentiel.

Quand la sonde effectue la sélection des charges en énergie, nous devons vérifier la relation (7) :

$$
\left(\frac{i}{I}\right)_{\text {sel }}=\frac{e v_{\mathrm{S}_{1}}}{K T_{\varepsilon}} .
$$

Nous avons d'abord vérifié qu'elle ne dépend pas de la fréquence, puis pour une fréquence constante de $230 \mathrm{~Hz}$, nous avons fait varier l'amplitude du signal de $20 \mathrm{mV}$ à $200 \mathrm{mV}$ crête à crête.

Nous remarquons que la courbe $I_{\mathrm{s}}=f\left(V_{\mathrm{S}}\right)$ est indépendante de $v_{\mathrm{S}_{1}}$ et que l'amplitude $i$ augmente proportionnellement à $v_{\mathrm{S}_{1}}$ quand $20<v_{\mathrm{S}_{1}}<100 \mathrm{mV}$ (fig. 7-8). Dans le cas où $e v_{\mathrm{S}_{1}}<K T_{\varepsilon}$, $\overline{I_{\mathrm{S}}}$ et $I_{\mathrm{S}_{0}}$ sont confondus. A partir de $200 \mathrm{mV}$, les hypothèses de la théorie ne sont plus valables, nous avons une déformation de $I_{\mathrm{S}}=f\left(V_{\mathrm{S}}\right)$ comme l'ont observée Boshi et Magistrelli [11] et la variation de $i$ n'est plus linéaire par rapport à $v_{\mathrm{S}_{1}}$. Dans ce dernier cas, la relation $e v_{\mathrm{S}_{1}} \ll K T_{\varepsilon}$ n'est plus vérifiée. En effet, nous avons des températures électroniques de l'ordre de $3600 \mathrm{~K}$, ce qui fait que la limite théorique pour $v_{\mathrm{S}_{1}}$ est de l'ordre de $300 \mathrm{mV}$.

De plus, nous trouvons avec la relation (7) une température de $3600 \mathrm{~K}$, valeur comparable à celle obtenue par la pente de la droite $\log I_{\mathrm{S}}$ et $\log i$ quand la sonde effectue la sélection des charges en énergie. 


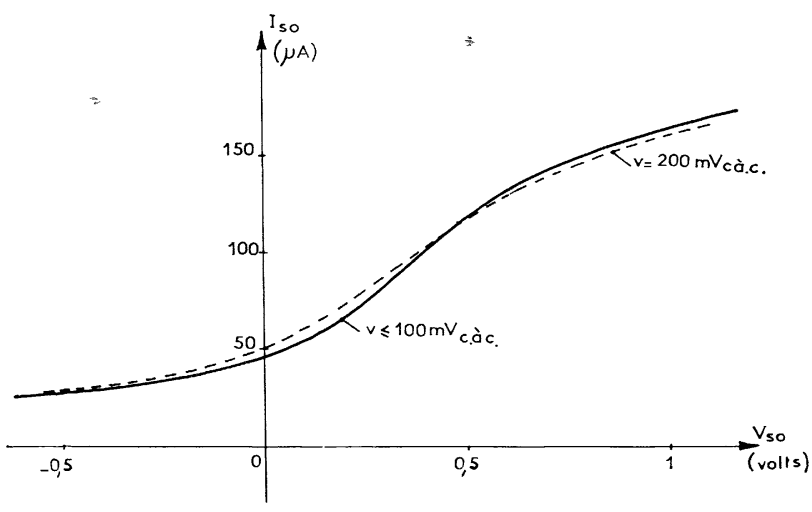

FIG. 7. - Variation de $I_{\mathrm{S}_{0}}$ dans le cas d'une simulation d'oscillations de potentiel.

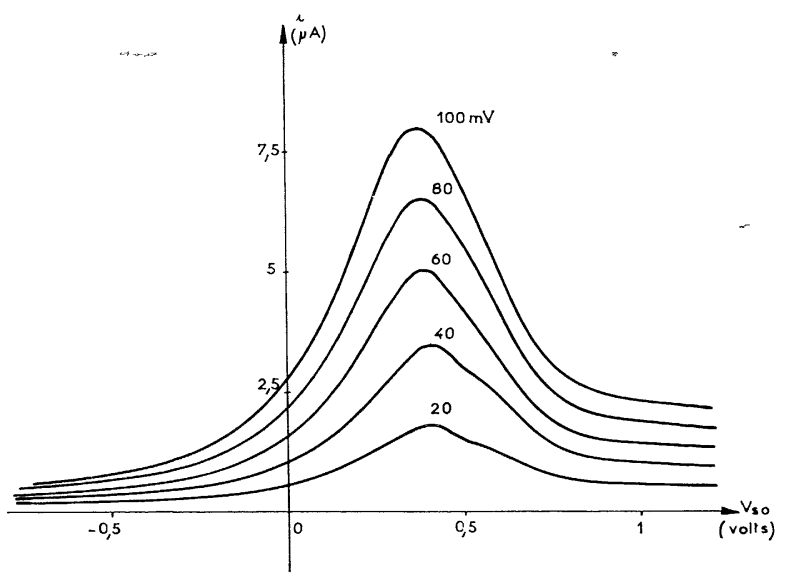

FIG. 8. - Variation du courant électronique fluctuant en fonction de l'amplitude $v_{\mathrm{S}_{1}}$ dans le cas d'une simulation d'oscillations de potentiel.

Des résultats analogues sont obtenus avec les caractéristiques ioniques : ainsi la figure 9 a donne la variation du courant ionique moyen $I_{\mathrm{S}_{0}}$ en fonction de la tension, variation qui, comme nous l'avons vérifié, est indépendante de la fréquence. La figure $9 \mathrm{~b}$ permet de suivre l'évolution du courant fluctuant en fonction de la tension de sonde pour différentes amplitudes du signal de perturbation. Nous déduisons là aussi la proportionnalité du courant fluctuant avec $v_{\mathrm{S}_{1}}$. Les valeurs de la température trouvée par les différentes méthodes exposées pour les électrons est de l'ordre de $1600^{\circ} \mathrm{K}$.

Dans le cas d'une simulation de fluctuation de potentiel par superposition d'un petit signal sur la tension de grille, nos hypothèses sur le fonctionnement de la sonde à grille immergée dans un plasma oscillant sont vérifiées de manière satisfaisante, compte tenu des difficultés expérimentales.

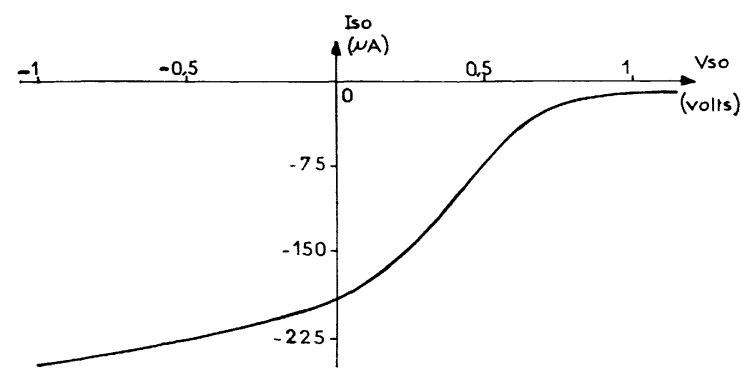

a

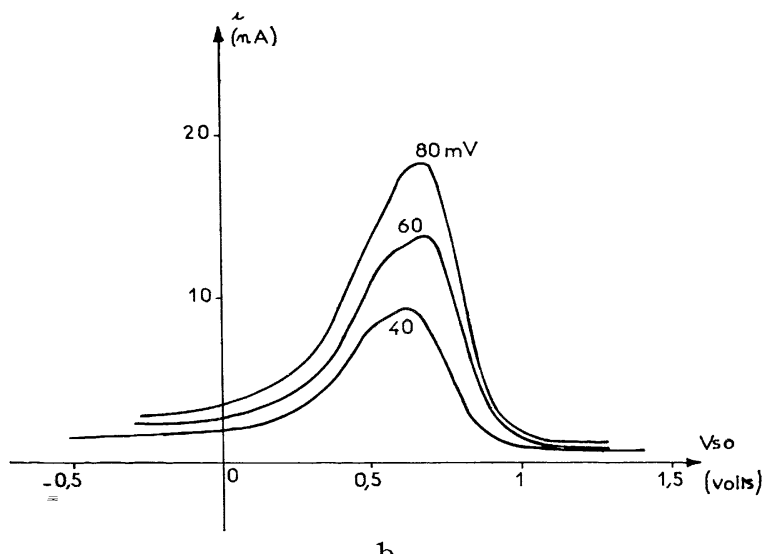

FIG. 9. - Variation du courant ionique dans le cas d'une simulation d'oscillation de potentiel de plasma :

a) Courant moyen.

b) Courant perturbé.

Oscillations de densité et de potentiel. - Dans un plasma calme, nous provoquons une oscillation de densité et de potentiel en perturbant localement le milieu au moyen d'une grille en tungstène placée devant la cathode chaude, un potentiel fluctuant sinusoïdal lui est appliqué $(40 \mathrm{mV}$ c à c, $2 \mathrm{kHz})$. Nous observons, à l'aide d'une sonde à grille, le comportement du plasma. Rappelons que l'amplitude du courant sinusoïdal peut s'exprimer par la relation :

$$
i=S e N_{0} v_{\mathrm{S}_{1}} g^{\prime}\left(V_{\mathrm{S}_{0}}\right)+\operatorname{Sen}_{1} \mathrm{e}^{j \varphi} g\left(V_{\mathrm{S}_{0}}\right) .
$$

La différence fondamentale entre cette expérience et celle de simulation d'oscillations de potentiel ( fig. 6 a et $6 \mathrm{~b}$ ) concerne l'existence d'un important courant fluctuant d'amplitude $i$ quand la sonde collecte les électrons à travers leur propre charge d'espace, comme le montre la figure 10. Nous notons, en effet, que le rapport des amplitudes $i$ des courants à saturation, dans la zone de sélection, est de $\frac{i_{\text {sat }}}{i_{\text {sel }}} \simeq 0,3$ dans le cas de la figure 6 (simulation d'oscillation de potentiel) et $\frac{i_{\text {sat }}}{i_{\text {sel }}} \simeq 0,8$ dans le cas de la figure 10 . Ceci peut être attribué à l'existence d'une perturbation dans la 


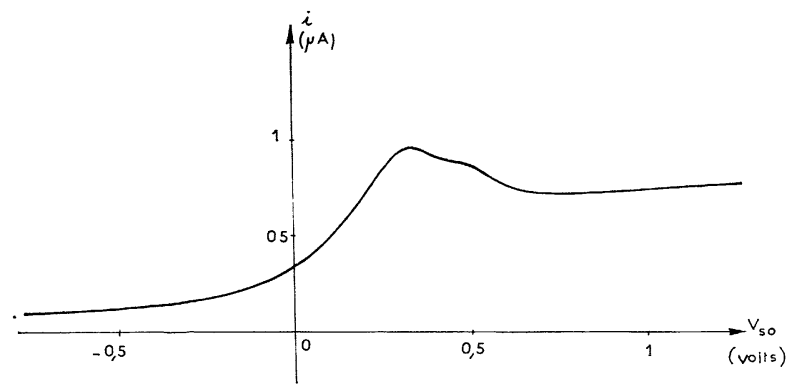

Fig. 10. - Cas d'oscillations simultanées de densité et de potentiel. Courbe expérimentale donnant l'amplitude résultante $\mathrm{du}$ courant fluctuant en fonction de la tension de sonde.

répartition de la densité de particules chargées dans le plasma. Nous observons également une variation de la phase du courant oscillant en fonction du potentiel appliqué à la sonde. Dans le cas d'une simulation de potentiel, nous n'observions pas ce phénomène de variation de la phase avec la tension $V_{\mathrm{S}_{0}}$. Ceci confirme l'hypothèse selon laquelle deux signaux déphasés l'un par rapport à l'autre se composent : l'un dépend des oscillations de densité, l'autre des oscillations de potentiel. Avec un détecteur synchrone, nous pouvons mettre en évidence de façon approximative la contribution respective des oscillations de densité et de potentiel au courant $i$ total ( $f g$. 11).

Sur la courbe 1, figure 11, nous avons la contribution des oscillations de densité.

Sur la courbe 2, figure 11, nous avons la contribution des oscillations de potentiel.

Les courbes 3 , figure 11 , sont semblables à la figure 9 et donnent l'amplitude résultante.

Des premières mesures, nous déduisons une valeur approchée du déphasage relatif, soit $\varphi \simeq 130^{\circ}$, valeur qui semble être due à l'importance des colli-

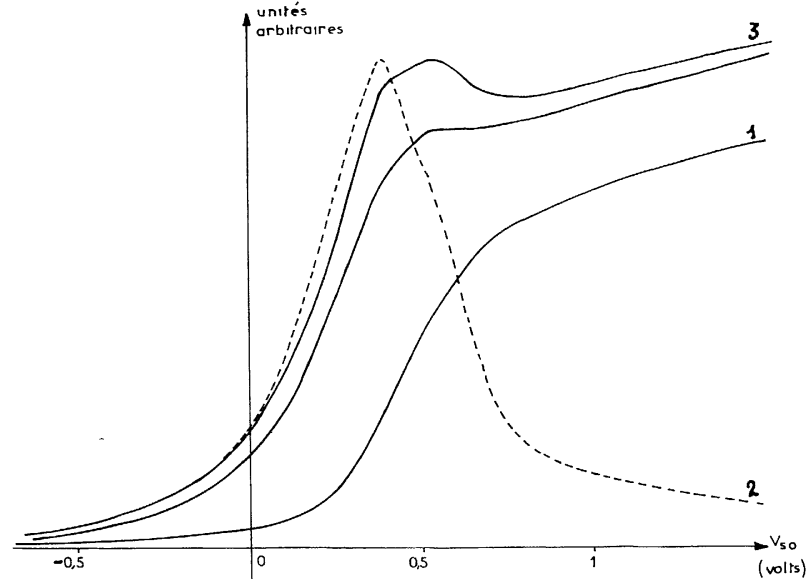

FIG. 11. - Décomposition de l'amplitude du courant fluctuant collecté par la sonde en ses deux composantes : l'une proportionnelle aux oscillations de densité, l'autre aux oscillations de potentiel.

sions dans le plasma. Une étude plus détaillée de la méthode de mesure du déphasage est exposée dans la référence [10].

Conclusion. - Nous avons établi une méthode permettant l'étude des fluctuations de densité et de potentiel local dans un plasma soumis à des perturbations d'origine propre ou externe. Les résultats de la théorie semblent confirmés par l'expérience et une extension est possible pour l'étude d'autres types de perturbations (ondes acoustiques ioniques dans un gaz faiblement ionisé). Il reste cependant une difficulté en ce qui concerne la mesure du déphasage relatif entre les paramètres fluctuants quoique nos premiers résultats semblent en accord avec les prévisions.

\section{BIBLIOGRAPHIE}

[1] Langmuir (I.), Tonks (L.) et Mot'T SMith (H. M.), Phys. Rev., 1926, 28, 104.

[2] FeldDen (M.), Haug (R.) et SchirmanN (D.), C. R. Acad. Sci. Paris, 1967, 264 B, 1243.

[3] HaUG (R.), FeldDeN (M.) et SchirmanN (D.), J.A.P., 1968, 39, 4501.

[4] Schirmann (D.), Thèse de spécialité, Nancy, 1968.

[5] Langmuir (I.) et MotT Suith (H. M.), Phys. Rev., 1926, 28, 4 .

[6] Crawford (F. W.), J.A.P., 1963, 34, 1897.
[7] Felden (M.), Patou (C.) et Haug (R.), Rev. Phys. Appl., 1966, 1, 48.

[8] Felden (M.), Haug (R.) et Schirmann (D.), J.A.P., 1967, 38, 2853.

[9] Haug (R.), L'onde électrique, 1966, 46, 1251.

[10] Cussenot (J. R.) et Haug (R.), C. R. Acad. Sci., 1969, 269 B, 253.

[11] Boshi et Magistreli, Il Nuovo Cimento, 1963, XXIX, 487. 